Check for updates

Cite this: Chem. Commun., 2020, 56,13888

DOI: $10.1039 / \mathrm{d} 0 \mathrm{cc} 90439 \mathrm{~b}$

rsc.li/chemcomm

\section{Retraction: Upconversion luminescent logic gates and turn-on sensing of glutathione based on two-photon excited quantum dots conjugated with dopamine}

\author{
Richard Kelly
}

Retraction of 'Upconversion luminescent logic gates and turn-on sensing of glutathione based on twophoton excited quantum dots conjugated with dopamine' by Rijun Gui et al., Chem. Commun., 2014, 50, 14847-14850, DOI: 10.1039/C4CC06181K.

(1) The Royal Society of Chemistry has been notified by the Office of Academic Research, Qingdao University that the authorship, affiliations and acknowledgements of this paper are incorrect. They informed us that "Rijun Gui has confirmed that he independently completed the experimental research before he joined Qingdao University. Without their knowledge and prior notice, he signed the names of irrelevant researchers (Hui Jin, Xifeng Liu, Zonghua Wang, Jianfei Xia, Min Yang, Feifei Zhang and Sai Bi) in his paper and added the Funding numbers of the irrelevant researchers in his papers without authorization. Rijun Gui confirmed that these irrelevant researchers did not participate in experimental researches reported in his paper and they did not provide financial support. They have no contribution to the paper." They concluded that "the names of the irrelevant researchers without contribution are required to be deleted from the authorship of the paper, and their funding numbers without providing financial support are required to be deleted from the "Acknowledgements"."

The corrected authorship list and affiliations for this paper are as follows:

Rijun Gui*a

${ }^{a}$ School of Chemistry and Chemical Engineering, Shanghai Jiao Tong University, Shanghai 200240, P. R. China.

Email: guirijun@163.com

The corrected Acknowledgements for this paper are as follows:

This work was financially supported by the Postdoctoral Science Foundation of Shanghai (13R21413800).

(2) The Royal Society of Chemistry hereby wholly retracts this Chemical Communications article due to concerns with the reliability of the data in the published article.

The TEM image in Fig. S1a duplicates data in a number of other publications, but reported as different materials. ${ }^{1-5}$

Given the significance of the concern about the validity of the data, the findings presented in this paper are no longer reliable. Rijun Gui requested to retract this article due to the incorrect authorship, but opposes the wording in this retraction notice. Signed: Richard Kelly, Executive Editor, Chemical Communications

Date: 21 st September 2020

\title{
References
}

1 R. Gui and X. An, RSC Adv., 2013, 3, 20959-20969.

2 Z. Xu, Z. Wu, J. Sun and R. Gui, Mater. Chem. Phys., 2015, 162, 286-290.

3 R. Gui, A. Wan, Y. Zhang, H. Li and T. Zhao, RSC Adv., 2014, 4, 30129-30136.

4 R. Gui, A. Wan, X. Liu and H. Jin, Chem. Commun., 2014, 50, 1546-1548.

5 H. Jin, R. Gui, J. Sun and Y. Wang, Talanta, 2018, 176, 277-283 (Retraction published 12 Jul 2019, Talanta, 2019, 204, 882). 